\title{
Los esquemas de rentas mínimas en Europa y el Ingreso Mínimo Vital (IMV)
}

\author{
Lluis Francesc Peris-Cancio \\ Sapienza Università di Roma, Italia \\ lluisfrancesc.periscancio@uniroma1.it
}

\section{INTRODUCCIÓN}

El siempre osado y premonitorio Bauman observa la pobreza en su dimensión más sociológica. En su libro Work, consumerism and the new poor (1998) se pregunta la dificil y nada banal cuestión de cómo se hace pobres a los pobres, cómo se llega a verlos como tales; hasta qué punto el modo de hacerlos o verlos depende de la manera en que nosotros (la gente común, ni ricos ni pobres) vivimos nuestra vida y elogiamos o despreciamos la forma en que otros lo hacen. Un punto de vista que contempla las personas empobrecidas no como como una realidad en sí, sino como la cristalización de procesos que implican a la sociedad entera.

Con esta perspectiva, la lucha para que nadie viva empobrecido, aun cuando necesita de un amplio análisis interdisciplinar, reclama una particular atención por parte de la sociología. Ante la mirada que distintas disciplinas, dominadas fundamentalmente por economistas y politólogos, han desarrollado sobre los esquemas de las rentas mínimas, la sociología aporta su visión: no se trata de encontrar el tratamiento, las rentas mínimas, para una disfunción, la pobreza. Consiste más bien en analizar los mecanismos que generan exclusión y considerar las propuestas de (re)distribución de la riqueza en todas sus dimensiones (material, cultural, relacional, etc.).

También se observa que el estudio de los instrumentos para luchar contra la pobreza está interesando a un número cada vez mayor de áreas de especialización sociológica. Esta evolución hacia una mayor transversalidad en la reflexión se refleja en la variedad de los temas tratados en los artículos seleccionados: el trabajo, la pobreza educativa, la igualdad de género, las migraciones o el capability approach, entre otros.

Es necesario que, ante un tema de tanta actualidad como el IMV, la sociología pueda dar su contribución específica con los instrumentos que le son propios, y ofrecer lecturas lo más posible objetivas basadas en el análisis empírico. Se agradece la disponibilidad que la Revista Española de Sociología ha brindado en la acogida de este debate, con el convencimiento que generará interés en estudiosos y policy makers. En los siguientes puntos se ofrece un encuadramiento preliminar del objeto de estudio y se ilustran algunos de los debates que se generan en torno a él. 


\section{Esquemas de rentas mínimas y nuevas formas de pobreza.}

Los esquemas de rentas mínimas (minimum income schemes), en adelante MIS, son objeto de un creciente interés académico y político, al ser entendidos como una medida clave en la lucha contra la pobreza y la exclusión. Al mismo tiempo, como subrayan los estudios de Marx y Nelson (2013), viven una continua evolución en su concepción y en su concreción práctica, por lo que la publicación de estudios específicos es contínua. Para Natili (2019), los MIS se caracterizan por un hecho paradójico que hoy parece más vigente que nunca: son sentidos como programas necesarios, especialmente durante periodos de crisis económica y, a la vez, se ven condicionados por las líneas de austeridad y contracción del gasto público que corresponden a un ciclo de desaceleración de la producción.

Un tema central en la reflexión sociológica sobre los MIS es el estudio del concepto mismo de pobreza y la identificación de sus nuevas formas, así como la descripción de las causas que llevan a personas a necesitar de una ayuda económica por parte del Estado. Como sostiene Saraceno (2015), el contexto socioeconómico de las actuales sociedades postindustriales alimenta niveles significativos de desigualdad, y deja en situación de vulnerabilidad a un número de personas que, en el imaginario social, no responden a la idea tradicional de "pobres"; en la medida que crece el trabajo atípico, la precariedad laboral, los bajos salarios, los working poor, el paro de larga duración y la falta de oportunidades para los jóvenes, se amplía el número de población empobrecida, que constituyen un nuevo desafio para las políticas públicas en su función de preservar la cohesión social.

Para hacer frente a esta tendencia, Noguera (2017) propone la articulación de acciones preventivas para reducir el número de personas y familias en esta situación. Plantea el concepto de predistribución, entendido como el instrumento que evita tener que destinar cuantiosos recursos en forma de garantía de rentas para la población en situación de pobreza, favoreciendo la introducción de una distribución primaria de la renta disponible que minimice el volumen de población pobre. Entre las medidas de predistribución se encuentran la dignificación del salario mínimo, una regulación del mercado de trabajo que contrarreste las tendencias a la precarización, el combate contra la pobreza laboral y una política de vivienda que impida que los precios de los alquileres absorban cualquier mejora en la garantía de rentas en las grandes ciudades.

\section{La agenda europea y la lucha contra la pobreza.}

La necesidad de medidas urgentes contra la pobreza y la exclusión ha activado la agenda política europea, de modo particular tras la crisis económica y financiera que se inició en el 2007. El protagonismo significativo en la reflexión y en el diseño de estrategias para paliar la situación ha visto a la UE en el centro de propuestas concretas. Una de las iniciativas más importantes ha sido el plan Europa 2020 (A Strategy for Smart, Sustainable and Inclusive Growth), adoptado en 2010, y cuyas orientaciones han influido en la determinación de las políticas nacionales de los países miembros. Con este se fija un objetivo común en la lucha contra la pobreza y la exclusión social, concretamente, la reducción de un $25 \%$ del número de europeos que viven por debajo del umbral de pobreza, cuya estimación se establece en 20 millones. En este periodo se constituye también la Plataforma Europea contra la Pobreza y la Exclusión Social, con la finalidad de analizar las políticas, optimizar el uso de los fondos de la UE, evaluar las prácticas, favorecer la innovación y generar una mayor coordinación entre las políticas de los países miembros. 
En el año 2013, ante la persistencia del número de personas en riesgo de pobreza, la UE propone un documento fundamental en el establecimiento de una línea común que conjugue desarrollo y cohesión: la comunicación Towards Social Investment for Growth and Cohesion - Social Investment Package. Así, la Comisión daba otro paso importante en la definición de un modelo de crecimiento inclusivo y social, instando a los Estados miembros a dar prioridad a la inversión en las personas, con una atención especial a la promoción de la infancia. También en este periodo nace una iniciativa europea, quizás menos conocida para el público en general, pero muy importante para los académicos, que se convertirá en un instrumento importante para el análisis sociológico: la elaboración del social scoreboard. Un cuadro de indicadores sociales que permite realizar de modo unívoco análisis comparativos, y que consiente alimentar un correcto benchmarking en la definición de las políticas de cada estado en la lógica del consolidado open method of coordination (OMC). El cuadro de indicadores se incluye en el Joint Employment Report of the Annual Growth Survey (AGS), que permite establecer las prioridades estratégicas de las políticas. En el 2020, comprobada la utilidad de este instrumento, se ha renovado y se ha pasado a llamar ASGS (Annual Sustainable Growth Survey) integrando los Sustainable Development Goals (SDGs) de las Naciones Unidas, incluyendo los objetivos de no pobreza, hambre cero y reducción de las desigualdades.

En sintonía con estas prioridades de la agenda política europea, y en particular con los principios del Social Investment Package, los países han realizado un esfuerzo para consolidar sistemas inclusivos de protección y bienestar que se sustenten en los tres pilares de la inclusión. Por un lado, el desarrollo de prestaciones de renta mínima adecuadas que permitan a los beneficiarios mejorar su condición e invertir en la de sus familias. En segundo lugar, la implementación de políticas activas que fomenten una inserción laboral real, concentrada en la empleabilidad de los más desfavorecidos. Por último, la consolidación de servicios territoriales de calidad que favorezcan y acompañen la inclusión social a largo plazo, comenzando por compensar las necesidades de los más vulnerables. La combinación de estos tres ejes de acción, fuertemente interrelacionados, ha comportado un renovado interés por los programas de rentas mínimas, por la exploración de sus posibilidades y por una renovada inversión en la mejora de su diseño. Como observa Hemerijck (2013) los MIS han vivido en este periodo un impulso a la evaluación y a la (re)conversión para transformarse en instrumento dinámico de promoción y activación que combine las prestaciones monetarias con una enérgica acción promocional a través de servicios cualificados para la (re)integración en el mercado laboral.

\section{El debate sobre los minimum income squemes}

Hay que recordar que estas iniciativas de inversión en programas de rentas mínimas no siempre han sido acogidas pacíficamente. Aunque la lucha contra la pobreza sea asumida como un objetivo que cuenta con el consenso de la mayor parte de las fuerzas políticas, el hecho de que personas y familias puedan recibir un beneficio económico que no proviene directa o indirectamente del propio trabajo ha sido objeto permanente de feroces críticas provenientes de las ideologías de matriz liberal. Anteponen el paradigma del workfare (o work first) con la insistencia en la necesidad de superar la protección económica con el incentivo (o la obligación) de la inserción laboral inmediata (Martínez-Mirto, 2019). Implícitamente, subyace una sospecha y un descrédito de las personas paradas de larga duración que benefician de ayudas, a las que se les atribuye falta de responsabilidad o de motivación. Este modelo fundamenta la intervención social como formación en habilidades básicas muy orientadas a responder a la oferta del mercado, con el objetivo de que la 
inserción laboral se concrete en el tiempo más breve posible, independientemente de la calidad de los puestos de trabajo o de su adaptación a las características de las personas demandantes de empleo, como han eficazmente descrito Carter y Whitworth (2017) en su análisis de la experiencia en Reino Unido.

Hay que tener en cuenta que el paradigma del social investment package también se orienta a la mejora de la empleabilidad de las personas mediante la recualificación profesional, pero con la condición de que la adquisición de competencias y la búsqueda de oportunidades de inserción esté orientada a soluciones duraderas y con un mínimo de calidad. Metodológicamente, se apuesta más por una intervención intensa, integral y adaptada a cada situación, centrada en la persona, no sometida a resultados en tiempo breve, con una lógica de inversión en el empoderamiento, acompañada en un proceso en el que la empleabilidad sea uno de los componentes en la adquisición de competencias amplias, y teniendo en consideración la influencia de una pluralidad de factores como edad, género, cargas familiares, salud, background cultural, etc.

Estas dos sensibilidades han conformado el debate principal por lo que se refiere a los esquemas de rentas mínimas en el contexto europeo, si bien, observando concretamente las políticas, parece que se camine hacia un progresivo nivel de convergencia. Es posible que esto no nazca tanto de un acuerdo de principios, sino más bien de una mayor consciencia para reconocer los límites de cada paradigma en su aplicación concreta: ni la pobreza se resuelve con la sola inversión en capital humano para el mercado, y los datos son en este sentido concluyentes, ni deja de existir un cierto riesgo de asistencialismo si no se favorece en las personas beneficiarias de las ayudas la consecución de una real emancipación de los sistemas de protección.

Superando lecturas generales o demasiado ideológicas, los estudios sobre la arquitectura institucional y la evolución de los MIS en este periodo (Frazier y Marlier, 2016; Marchal y Van-Mechelen, 2017) han permitido identificar con mayor detalle los mecanismos internos a los programas. Así, a través de un análisis de la realidad empírica, muestran evidencias de la eficacia en la reducción de los niveles pobreza y exclusión, y, en líneas generales, confirman la necesidad de una integración entre distintos niveles de las políticas sociales.

\section{Características y evolución de los casos precedentes del IMV europeos}

La ideación del IMV se ha beneficiado de la lectura de las experiencias que otros países europeos han producido en el desarrollo de esquemas de rentas mínimas. Seguramente, todos ellos tienen en común algunas características definitorias: han sido concebidos como instrumentos de lucha contra la pobreza del tipo (last) social safety nets, un último recurso selectivo para la población que demuestra una situación de necesidad económica (means-test) y se activan a iniciativa del interesado/a cuando se ha agotado la posibilidad de beneficiarse de cualquier otro amortiguador social o cuando se comprueba que no son aplicables otras medidas económicas de protección social en favor de la persona (Busilacchi, 2018). Se concreta en una trasferencia económica dedicada a la unidad de convivencia, calculada en función de la diferencia entre los ingresos efectivos de esta y un umbral de referencia determinado en cada contexto. La cantidad del beneficio depende normalmente también del número de miembros de la unidad de convivencia, así como de otros factores que el legislador puede incluir para su cálculo, como patrimonio, casa, etc. En la mayoría de los países europeos, las cuantías no son suficientes para ofrecer un nivel de protección adecuado (Cantillon et al., 2017). Por otro lado, los MIS difieren en su diseño según numerosas variables: cantidad de la ayuda, duración, condicionalidad, compatibilidad con ingresos por trabajo, ayudas extras para la vivienda, atención a las 
cargas familiares, obligaciones vinculantes, etc. En cada ley de implantación de rentas mínimas se definen estas variables, que, según el periodo histórico, tienden a orientarse en un modo o en otro.

No por casualidad, los primeros programas de rentas mínimas que aparecieron tras la segunda guerra mundial estaban caracterizados por una extrema residualidad respecto a los mecanismos contributivos de la seguridad social de inspiración bismarkiana, presentes en muchos casos ya desde finales del siglo anterior; son una excepción a esta concepción del welfare continental esquemas más generosos nacidos en los años cincuenta y sesenta en estados como Finlandia, Suecia y Países Bajos.

Una segunda fase aparece con la crisis petrolífera de los años setenta. El aumento del paro estructural deja a la luz las lagunas de los sistemas de protección social desarrollados en el periodo de boom industrial, obligando a adoptar esquemas de rentas mínimas como pilares de la política social. La desocupación, y, a la vez, las dificultades en la sostenibilidad de los presupuestos de los estados llevan a una tercera fase en los años ochenta y que se caracteriza, por un lado, en la necesidad de contener el gasto público, y, por otro, en la introducción de la condicionalidad vinculada a la disponibilidad inmediata a la inserción laboral. En este periodo comienza también a ser relevante el peso institucional de la Unión Europea, que ejercita un rol específico en el intento de acompañar y dar orientaciones a los estados de bienestar europeos en el esfuerzo común de lucha contra la exclusión social.

Con la llegada de la crisis económica del 2007, aparece una recesión económica sin precedentes con el consecuente aumento de la pobreza. Esto supuso una prueba de resistencia inesperada: en primer lugar, los MIS sobrellevaron un aumento exponencial en el número de beneficiarios, y, por otro, los mecanismos de protección contributivos de la seguridad social se agotaron sin que el mercado hubiera podido absorber trabajadores que pasaron a una situación de fuerte dificultad económica sin recibir protección alguna.

La emergencia producida por el aumento de las situaciones de pobreza tuvo sus consecuencias: a corto plazo llevó a la introducción de medidas accesorias, a menudo temporales, para aumentar la cobertura y la generosidad de los MIS en algunos contextos; a medio plazo se observó un sucesivo endurecimiento de los criterios de acceso y de contención de los gastos en la mayoría de los Estados miembros (Marchal et al., 2016). La segunda innovación de esta cuarta fase en Europa se refiere a la aceleración en materia de MIS de países pertenecientes al welfare mediterráneo que no contaban con ellos y que con mayor fuerza habían sufrido los efectos de la crisis. Las dificiles circunstancias han conducido a la superación del retraso histórico en Grecia e Italia, que, con las primeras señales de recuperación económica, han introducido esquemas estables de rentas mínimas a escala nacional en el 2017 y 2018 , respectivamente.

Mirando al futuro, bien podría ser que una quinta etapa de este proceso de los MIS se corresponda con el establecimiento de una renta mínima para todos los ciudadanos europeos, que podría concebirse como un suelo común a integrar en cada contexto nacional, y que podría ser gestionada con un método abierto de coordinación. Con los primeros debates dirigidos por la Presidencia alemana, retomados en la sesión del EPSCO del 13 de octubre 2020, la idea comienza a tomar cuerpo lentamente, empujada por la emergencia social consecuencia de la crisis sanitaria actual, común en mayor o menor medida a todos los países europeos, como se recoge en el documento Strengthening Minimum Income Protection to Combat Poverty and Social Exclusion in the COVID-19 Pandemic and Beyond (Council of the European Union 2020). 


\section{La introducción del Ingreso Mínimo Vital en España.}

El Real Decreto-ley 20/2020, con el que se ha establecido el IMV, es sin duda un hito en las políticas sociales del Estado español, y un paso adelante sin precedentes en la lucha contra la pobreza y la exclusión social, o si se prefiere, en la reducción de las desigualdades sociales en España. Madurado en un largo proceso de elaboración, fruto de la activación de la sociedad civil y del debate político en el contexto de la postcrisis del crack económico del 2007, la irrupción de la inesperada calamidad sanitaria ha acelerado su nacimiento como medida no sólo estructural, sino también contingente a una emergencia social acuciante tras la declaración del estado de alarma.

Aunque los trámites parlamentarios para el establecimiento del IMV se hayan realizado velozmente y haya sido aprobada por una amplísima mayoría parlamentaria, su introducción y su sucesiva puesta en marcha no ha dejado de ser objeto de un encendido debate: por una parte, desde una perspectiva más conservadora, por las acusaciones de ser una política cara e ineficaz que estimula la pasividad de los beneficiarios; por otra, desde contextos progresistas, aparece una nueva visión crítica por la que se percibe el IMV, y todos los esquemas de rentas mínimas, como un intento fallido de alcanzar una propuesta más ambiciosa e igualitaria de Renta Básica Universal. Además, teniendo en cuenta la configuración del Estado español, la introducción del IMV como una prestación «suelo» de carácter nacional, ha comportado que exponentes de algunas comunidades autónomas lo consideren una impropia recentralización de competencias, dificilmente integrable con las medidas de inclusión activa reservadas al nivel autonómico.

Es cierto que el IMV nace condicionado por decenios de una variada y rica experiencia de rentas mínimas autonómicas en España, un panorama que ofrece luces y sombras. Como emerge de los trabajos de Bergantiños, Quiles y Bacigalupe (2017); de Ayala (2016) y de Ayala, Arranz, García-Serrano y Martínez Vitro (2020), la experiencia de las distintas regiones muestra una heterogeneidad en las coberturas. Además, aparece una correlación con el PIB de cada región, de modo que, paradójicamente, las regiones más ricas proporcionan una mejor cobertura, mientras que las regiones más pobres el contrario, llegando incluso a suspender sus esquemas de rentas durante el periodo de gran recesión económica a causa de sus limitaciones presupuestarias. De ahí que la implementación del IMV propone un reto dificil: construir una medida nacional de garantía de prestación mínima para todos los ciudadanos sin alterar las prácticas de excelencia ya consolidadas a nivel regional. Es por ello que, para su estudio y valoración, sea oportuno tener separados dos conceptos: la concepción en sí del IMV como MIS nacional, y, por otro lado, la modalidad de implementación que progresivamente establezca en acuerdo con los gobiernos autonómicos.

\section{Diseño y caracterización del Ingreso Mínimo Vital.}

La configuración a la base del IMV responde a una serie de características que lo sitúan en línea con los MIS europeos más avanzados, salvo por cuanto se refiere al alcance de las trasferencias económicas que ofrece, claramente inferior a esquemas más generosos del norte de Europa. Entre sus elementos más destacados se pueden destacar tres: su concepción como derecho subjetivo permanente, la gobernanza multinivel y los instrumentos de activación.

A diferencia de muchos precedentes autonómicos, el IMV se define como una renta garantizada que adquiere el rango de derecho subjetivo, evitando depender de los presupuestos anuales y de eventuales cambios en las orientaciones de los gobiernos, quedando introducido como una nueva prestación económica en el sistema de Seguridad 
social de carácter indefinido, no contributivo, financiada con transferencias derivadas de la Ley de Presupuestos Generales del Estado y vinculada sólo a una residencia legal en territorio nacional de al menos un año.

Se instituye como un suelo de protección para todos los residentes; teniendo en cuenta los MIS que ya existen en cada Comunidad Autónoma, el IMV pretende integrarse compatiblemente con los mismos, dentro de unos límites cuantitativos, respetando el margen de actuación de las políticas sociales de cada Comunidad Autónoma, a las que invita a procurar acciones de mejora. Establece reglas de coordinación y cooperación con Comunidades Autónomas y con los entes locales, de modo que los servicios territoriales de ambos niveles de la administración jueguen un papel relevante en la información y eventual gestión del IMV, así como en la orientación para el empleo. Es clave el acierto y el dinamismo con el que se determinará la conexión del ingreso mínimo con las políticas activas de empleo, especialmente en la formación y en la reinserción profesional, así como en los incentivos a la inclusión en el mercado de trabajo. En este sentido, queda al nivel autonómico la responsabilidad de gobernar espacios de sinergia y coordinación con el Servicio Público de Empleo Estatal en las políticas activas, a fin de introducir los programas y las iniciativas más eficaces en cada territorio (Ramos-Quintana, 2020). No se puede descartar que se produzca un efecto no deseado por el que se manifieste la tendencia a mantener ciertas diferencias que ya existían entre territorios.

Es inteligente y acorde con las recientes reformas en otros países europeos, el diseño para la activación de los beneficiarios y el modo de incentivar la incorporación al empleo. Por un lado, para la solicitud del IMV es necesario inscribirse como demandante de empleo, si es que no se figuraba en las listas anteriormente. Por otro lado, no se considera incompatible el beneficio con ulteriores prestaciones de trabajo con baja remuneración. El mecanismo propuesto calcula una correlación por la que, por cada euro ingresado en concepto de salario, se deduce una proporción menor en la cuantía del IMV, reservándole así su función de última garantía de ingresos, pero haciendo siempre conveniente acceder a una actividad laboral, aunque esta sea modesta o escasamente pagada. De este modo se evita la disyuntiva entre mantener la prestación o aceptar un trabajo, y se desincentiva el trabajo remunerado no declarado.

Por cuanto se refiere a su implementación, ya han sido señalados los primeros problemas en su gestión (baja cobertura, burocratización, brecha digital...). Aunque es previsible que este sea un aspecto delicado del IMV, se entiende que ha pasado poco tiempo desde que fuera publicada la ley, y que las circunstancias de su primer desarrollo han sido extremamente particulares por causa de la pandemia, por lo que es necesario esperar para extraer conclusiones.

\section{Elementos para un debate sociológico}

La introducción del IMV abre amplios espacios de debate desde distintas perspectivas y disciplinas. Se han seleccionado contribuciones de expertos que tratan con originalidad algunos temas de innegable interés sociológico: welfare state, capability approach, pobreza educativa, género y migraciones, considerados en la mayor parte de los casos, desde una perspectiva europea.

En el primer artículo de Ana Arriba González y Manuel Aguilar Hendrickson se ofrece una lectura de los precedentes que han llevado a la introducción de la medida, ilustrando con datos comparativos las modalidades de protección que han caracterizado al Estado español. Según el análisis, este instrumento mejora apreciablemente la cobertura asistencial, pero deja sin resolver la adecuación del conjunto a las nuevas formas de empleo. 
El artículo de Elisa Matutini reflexiona, desde el punto de vista del capability approach, sobre la relación entre la activación, la condicionalidad y el trabajo social en los esquemas de ingresos mínimos. Partiendo de una lectura de las experiencias europeas, considera que situar la trayectoria de las personas en el centro de la intervención supone pensar en un sistema que esté atento a las necesidades específicas y libre de los mecanismos de estandarización y burocratización de la intervención.

Luca Salmieri y Oracio Giancola amplían la envergadura del debate con un artículo en el que se subraya la importancia de asociar las intervenciones económicas de lucha contra la pobreza a las políticas de reducción de la pobreza educativa. Introducen la noción de doble responsabilidad de los gobiernos, que por un lado deben invertir en el apoyo económico a las familias, pero, por otro, también deben hacerlo en la educación y en el fortalecimiento de las habilidades básicas y de las políticas de alfabetización.

Una cuarta contribución al debate es ofrecida por Olga Salido Cortés, que se pregunta hasta qué punto la introducción del IMV ayudará a cerrar la brecha de género de la pobreza, la precariedad y la discriminación que sufren las mujeres. Concluye que esto dependerá en gran medida de que venga acompañada por medidas de carácter estructural, haciendo propias las dos cuestiones centrales de la agenda feminista: la reorganización de los cuidados y la autonomía financiera de las mujeres.

Por último, quien escribe, ofrece una lectura sobre la participación que las personas extranjeras podrían tener al IMV en base a un estudio comparado con experiencias de otros tres países europeos. El resultado es que la concepción del instrumento ayuda a que no se dé una discriminación de facto, pero que en gran medida dependerá de la contribución de los servicios territoriales de las comunidades autónomas a facilitar la compatibilidad de los procesos.

\section{CONCLUSIONES}

El IMV abre un nuevo capítulo en la lucha contra la pobreza en España. El contexto de crisis sanitaria ha acelerado su introducción en la agenda política. Nacido mucho después que la mayor parte de las experiencias de MIS en Europa, recoge en su concepción elementos que lo ponen, según ciertos criterios, a la vanguardia.

Menos clara es la implementación y la evolución que irá adquiriendo en los próximos años. Aparece como espacio de mayor incerteza la integración que se conseguirá con las medidas de cada comunidad autónoma, en especial por lo que hace referencia a los servicios sociales y para la inserción laboral. Conceptos tan relevantes en la lucha contra la pobreza como accesibilidad, activación, empleo, casa, etc., quedan supeditados a las modalidades que, en una lógica de complementariedad, puedan impulsarse desde las distintas regiones. De este hecho puede depender el impacto que la medida tenga entre los colectivos más vulnerables.

Por último, sería lógico que la filosofia y la finalidad del IMV cree un efecto desencadenante y venga acompañada de reformas en otros niveles de las políticas sociales, como por ejemplo en las medidas de protección contributivas, en la regulación laboral y el derecho a la casa; solo a través de esta armonización se podrán alcanzar mayor éxito en el propósito de promover una sociedad con un mayor nivel de igualdad. 


\section{REFERENCIAS}

Ayala, L. (2016). El gasto público en programas de lucha contra la pobreza: tendencias, determinantes y necesidades de reforma. Papeles de economía española, 147, 145-166.

Ayala, L., Arranz, J., García-Serrano, C., y Martínez-Virto, L. (2020). The effectiveness of minimum income benefits in poverty reduction in Spain [Early View]. International Journal of Social Welfare, . Recuperado de https://dx.doi.org/10.1111/ijsw.12447.

Bauman, Z. (1998). Work, consumerism, and the new poor. Berkshire: Mc Graw-Hill House.

Bergantiños, N., Quiles, R., y Bacigalupe, A. (2017). Las rentas mínimas de inserción en época de crisis. ¿Existen diferencias en la respuesta de las comunidades autónomas? Papers. Revista de Sociologia, 102(3), 399-399. Recuperado de https://dx.doi.org/10.5565/rev/ papers.2315.

Busilacchi, G. (2018). Le politiche di reddito minimo in Europa: un cambio paradigmatico verso una nuova convergenza? Sinappsi, 8(3), 84-93.

Cantillon, B., Marchal, S., y Luigjes, C. (2017). Decent Incomes for the Poor: Which Role for Europe? Journal of Common Market Studies, 55(2), 240-256. Recuperado de https://dx.doi.org/10.1111/jcms.12486.

Carter, E. y Whitworth, A. (2017). Work Activation Regimes and Well-being of Unemployed People: Rhetoric, Risk and Reality of Quasi-Marketization in the UK Work Programme. Social Policy \& Administration, 51(5), 796-816. Recuperado de https:/ / dx.doi.org/10.1111/ spol.12206.

Frazier, H. y Marlier, E. (2016). Minimum Income Schemes in Europe: A study of national policies 2015. Bruselas: European Commission.

Hemerijck, A. (2013). Changing Welfare States. Oxford: Oxford University Press.

Marchal, S., Marx, I., y Van-Mechelen, N. (2016). Minimum income protection in the austerity tide. IZA Journal of European Labor Studies, 5(4), 2-20.

Marchal, S. y Van-Mechelen, N. (2017). A New Kid in Town? Active Inclusion Elements in European Minimum Income Schemes. Social Policy and Administration, 51(1), 171-194. Recuperado de https://doi.org/10.1111/spol.12177.

Martínez-Mirto, L. (2019). Nueva pobreza, precariedad y rentas mínimas: respuestas para incentivar el empleo en el actual contexto sociolaboral. Cuadernos de Relaciones Laborales, 37(1), 155-175. Recuperado de https://doi.org/10.5209/CRLA.63824.

Marx, I. y Nelson, K. (eds.) (2013). Minimum income protection in flux. Londres: Palgrave MacMillan.

Natili, M. (2019). The Politics of Minimum Income in the Age of Austerity: An Introduction. En The Politics of Minimum Income. Work and Welfare in Europe (pp. 1-20). Londres: Palgrave Macmilan.

Noguera, J. A. (2017). Redistribución, predistribución y garantía de rentas. En Zalakaín, J. y Barragué, B. (coords), Repensar las políticas sociales: predistribución e inversión social (pp. 207-228), Editorial Grupo 5/Kutxa Fundazioa, Madrid. 
Council of the European Union. (2020). Strengthening Minimum Income Protection to Combat Poverty and Social Exclusion in the COVID-19 Pandemic and Beyond. Council Conclusions. Recuperado de https://data.consilium.europa.eu/doc/document/ ST-11721-2020-INIT/en/pdf.

Ramos-Quintana, M. I. (2020). El ingreso mínimo vital como instrumento para combatir la pobreza y la exclusión desde el sistema de la seguridad social. Revista Hacienda Canaria, (53), 295-319.

Saraceno, C. (2015). Il lavoro non basta. La povertà in Europa negli anni della crisi. Milán: Feltrinelli. 